\title{
Uniqueness of meromorphic functions concerning their difference operators and derivatives
}

Dong-Mei Wei ${ }^{1}$ and Zhi-Gang Huang ${ }^{1 *}$ (D)

\section{"Correspondence:}

alexehuang@sina.com

'School of Mathematics and

Physics, Suzhou University of

Science and Technology, Suzhou,

P.R. China

\section{Springer}

\begin{abstract}
Let $f$ and $g$ be two nonconstant meromorphic functions. Shared value problems related to $f$ and $g$ are investigated in this paper. We give sufficient conditions in terms of weighted value sharing which imply that $f$ is a linear transformation or inversion transformation of $g$. We also investigate the uniqueness problem of meromorphic functions with their difference operators and derivatives sharing some values.
\end{abstract}

MSC: 30D35; 39A05

Keywords: Meromorphic function; Difference operator; Shared values; Periodic function; Finite order

\section{Introduction and main results}

Throughout this paper, a meromorphic function is assumed to be meromorphic in the whole complex plane. The basic notations and results of Nevanlinna value distribution theory of meromorphic function are assumed to be known to the reader; see, e.g., [10, 21]. For a meromorphic function $f(z)$, we define the order and the lower order of $f(z)$ by

$$
\sigma(f)=\limsup _{r \rightarrow \infty} \frac{\log ^{+} T(r, f)}{\log r} \text { and } \mu(f)=\liminf _{r \rightarrow \infty} \frac{\log ^{+} T(r, f)}{\log r}
$$

while

$$
\lambda(f)=\limsup _{r \rightarrow \infty} \frac{\log ^{+} N\left(r, \frac{1}{f}\right)}{\log r} \text { and } \delta(a, f)=\liminf _{r \rightarrow \infty} \frac{m\left(r, \frac{1}{f-a}\right)}{T(r, f)}=1-\limsup _{r \rightarrow \infty} \frac{N\left(r, \frac{1}{f-a}\right)}{T(r, f)}
$$

stand for the exponents of convergence of zero sequence of $f$ and the deficiency of $f$ at the point $a$, respectively. For a nonconstant meromorphic function $h$, we denote by $T(r, h)$ the Nevanlinna characteristic of $h$ and by $S(r, h)$ any quantity satisfying $S(r, h)=o(T(r, h))$, as $r$ runs to infinity outside of a set $E \subset(0,+\infty)$ of finite linear measure. We say that $h$ is a small function of $f$ if $T(r, h)=S(r, f)$. In the following, we denote by $I$ a set of infinite linear measure not necessarily the same in all its occurrences.

(c) The Author(s) 2020. This article is licensed under a Creative Commons Attribution 4.0 International License, which permits use sharing, adaptation, distribution and reproduction in any medium or format, as long as you give appropriate credit to the original author(s) and the source, provide a link to the Creative Commons licence, and indicate if changes were made. The images or other third party material in this article are included in the article's Creative Commons licence, unless indicated otherwise in a credit line to the material. If material is not included in the article's Creative Commons licence and your intended use is not permitted by statutory regulation or exceeds the permitted use, you will need to obtain permission directly from the copyright holder. To view a copy of this licence, visit http://creativecommons.org/licenses/by/4.0/. 
We say that $f$ and $g$ share the value $a$ IM (ignoring multiplicities), if $f$ and $g$ have the same $a$ point. If $f$ and $g$ have the same $a$ point with the same multiplicities, then we say $f$, $g$ share the value $a \mathrm{CM}$ (counting multiplicities). Let $k$ be a positive integer, we denote by $N_{k)}\left(r, \frac{1}{f-a}\right)$ the counting function of $a$ points of $f$ with multiplicity $\leq k$ and by $N_{(k+1}\left(r, \frac{1}{f-a}\right)$ the counting function of $a$ points of $f$ with multiplicity $>k$, where each $a$ point is counted according to its multiplicity. Similarly we define $\bar{N}_{k)}\left(r, \frac{1}{f-a}\right)$ and $\bar{N}_{(k+1}\left(r, \frac{1}{f-a}\right)$ where in counting the $a$ points of $f$ we ignore the multiplicities.

Definition 1 ([12]) Let $k$ be a nonnegative integer or infinity. For $a \in \mathbb{C} \cup\{\infty\}$, we denote by $E_{k}(a ; f)$ the set of all $a$ points of $f(z)$ where an $a$ point of multiplicity $m$ is counted $m$ times if $m \leq k$ and $k+1$ times if $m>k$. If $E_{k}(a ; f)=E_{k}(a ; g)$, then we say that $f, g$ share the value $a$ with weight $k$.

We write $f$ and $g$ share $(a, k)$ to mean that $f, g$ share the value $a$ with weight $k$.

Definition 2 ([12]) If $s$ is a positive integer, then we denote by $N\left(r, \frac{1}{f-a} \mid=s\right)$ the counting function of those $a$ points of $f$ whose multiplicity is $s$, where each $a$ point is counted according to its multiplicity. For a positive integer $m$, denote by $N(r, a ; f \mid \geq m)$ the counting function of those $a$ points of $f$ whose multiplicities are not less than $m$ where each $a$ point is counted according to its multiplicity.

Definition 3 ([13]) Denote by $N_{2}\left(r, \frac{1}{f-a}\right)$ the sum of $\bar{N}\left(r, \frac{1}{f-a}\right)+\bar{N}\left(r, \frac{1}{f-a} \mid \geq 2\right)$.

The classical four point and five point theorems of Nevanlinna [15] show $f$ is a Möbius transformation of $g$ if two meromorphic functions $f$ and $g$ share four distinct values CM, and $f=g$ if $f$ and $g$ share five distinct values IM. The assumption 4CM of the fourpoint theorem and 5IM of the five-point theorem have been improved to 2CM+2IM and $3 \mathrm{CM}+1 \mathrm{IM}$, while $1 \mathrm{CM}+3 \mathrm{IM}$ remains an open problem.

Some researchers also considered whether the conditions of shared values can be replaced by other conditions. Ozawa [16] obtained the following.

Theorem A Let $f$ and $g$ be nonconstant entire functions of finite order such that $f$ and $g$ share 0 and 1 CM. If $\delta(0, f)>\frac{1}{2}$, then $f \cdot g=1$ or $f=g$.

Removing the order restriction Ueda [19] and Yi [22] obtained some improvements of Theorem A. Especially, Yi [23] obtained the following.

Theorem B ([23]) Suppose that $f(z)$ and $g(z)$ are nonconstant meromorphic functions. If $f, g$ share $0,1, \infty C M$ and $\bar{N}\left(r, \frac{1}{f}\right)+\bar{N}(r, f)<(d+o(1)) T(r, f)$ for $r \in I$ and $r \in \infty$, where $d$ is a positive number satisfying $0<d<\frac{1}{2}$, while $I \subset(0,+\infty)$ is a subset of infinite linear measure, then $f=g$ or $f \cdot g=1$.

For the sake of relaxing the nature of sharing of values and improving Theorem B, Lahiri in [12] obtained the following result in terms of the weighted value sharing. 
Theorem C ([12]) Suppose that $f(z)$ and $g(z)$ are nonconstant meromorphic functions. Let $f$ and $g$ share $(0,1),(\infty, 0),(1, \infty)$. If

$$
N\left(r, \frac{1}{f-a} \mid=1\right)+4 \bar{N}(r, f)<(d+o(1)) T(r, f),
$$

then either $f=g$ or $f \cdot g=1$.

Our first result shall follow this direction and investigate the uniqueness of meromorphic functions in terms of weighted value sharing.

Theorem 1.1 Let $f$ and $g$ be meromorphic functions with finite order, and let $c \in \mathbb{C} \backslash\{0\}$. Suppose that $f^{n}$ and $g^{n}$ share $(R(z), l)$, where $R(z)$ is a rational function and $l, n$ are integer. If one of the following cases holds:

(1) $l=0, n \geq 15$

(2) $l=1, n \geq 10$

(3) $l \geq 2, n \geq 9$,

then $f=\operatorname{tg}$ orf $\cdot g=t \alpha$, where $t^{n}=1, \alpha^{n}=R^{2}$.

Rubel and Yang [18] in 1977 initiated the study of entire functions sharing values with their derivatives instead of studying the problem of sharing value of two meromorphic functions $f$ and $g$.

Theorem $\mathbf{D}$ Let $f$ be a nonconstant entire function. If $f$ shares two distinct finite values $C M$ with $f^{\prime}$, then $f \equiv f^{\prime}$.

More results on the uniqueness of $f^{\prime}$ with its nth derivative $f^{(n)}$ were obtained by several authors; see $[1,8,20]$. In view of the progress on the difference analogues of classical Nevanlinna theory of meromorphic functions [4, 9], it is quite natural to investigate the uniqueness problems of meromorphic functions and their difference operators; see $[3,6,11,25]$. So a natural question arises, that is, how about the uniqueness of the derivatives and the difference operators of $f(z)$ ?

In 2018, Qi et al. [17] obtained some results in the case that $f^{\prime}(z)$ shares values with $\Delta f$ or $f(z+c)$.

Theorem $\mathbf{E}$ Let $f$ be a meromorphic function of finite order. Suppose that $f^{\prime}$ and $\Delta f$ share $a_{1}, a_{2}, a_{3}, a_{4} I M$, where $a_{1}, a_{2}, a_{3}, a_{4}$ are four distinct finite values. Then $f^{\prime}=\Delta f$.

Theorem $\mathbf{F}$ Let $f$ be a transcendental entire function of finite order, and a be a nonzero finite value. If $f^{\prime}(z)$ and $f(z+c)$ share $0, a C M$, then $f^{\prime}(z)=f(z+c)$ for all $z \in \mathcal{C}$.

Regarding Theorems E and F, one may ask the following question:

Question 1 What can be said when the difference operators of $f(z)$ shares some values with its derivatives?

In this direction, we will prove the following results. 
Theorem 1.2 Let $f$ be a nonconstant entire function of finite order with periodic $\eta \neq 0$ such that $\mu(f)>1$, where $\eta$ is a finite nonzero value, and let $a_{1}$ and $a_{2}$ be two distinct finite values, and $k$ be a positive integer. If $\Delta f$ and $f^{(k)}$ share $a_{1} C M, \Delta f$ and $f^{(k)}$ share $a_{2} I M$, then $\Delta f=f^{(k)}$.

If we replace $\Delta f(z)$ by the shift $f(z+c)$, we can obtain the following.

Theorem 1.3 Let $f$ be a nonconstant meromorphic function of finite order, and let $c$ be a finite nonzero value, let $k$ be a positive integer satisfying $k \geq 2$, and let $a_{1}, a_{2}, a_{3}$ be three finite values such that $a_{1} \neq 0, a_{2} \neq 0$ and $N\left(r, \frac{1}{f-a_{3}}\right)=S(r, f)$. If $f(z+c)$ and $f^{(k)}(z)$ share $a_{1}$ $C M$ and $a_{2} I M$, then $f(z+c)=f^{(k)}(z)$ for all $z \in \mathcal{C}$.

Remark The results obtained in this manuscript have many applications in the qualitative analysis of differential equations with piecewise constant arguments and porous medium problems; see [5, 14]. The following example shows that the assumption $\mu(f)>1$ of Theorem 1.2 is the best possible.

Example 1 Let $f(z)=e^{A z}$, where $A \neq 0$ is a constant. Then $f^{(k)}=A^{k} e^{A z}$ and $\Delta(f)=f(z+$ 1) $-f(z)=\left(e^{A}-1\right) e^{A z}$. Clearly, $\Delta(f)$ and $f^{(k)}$ share $0 \mathrm{CM}$ and $\infty \mathrm{IM}$, and that $\mu(f)=1$. We can choose $A$ such that $e^{A}-1 \neq A^{k}$, and so $f^{\prime} \not \equiv \Delta(f)$.

\section{Preliminaries}

First we denote by $H$ the following function:

$$
H=\left(\frac{F^{\prime \prime}}{F^{\prime}}-\frac{2 F^{\prime}}{F-1}\right)-\left(\frac{G^{\prime \prime}}{G^{\prime}}-\frac{2 G^{\prime}}{G-1}\right)
$$

where and in what follows, $F$ and $G$ are some two distinct nonconstant meromorphic functions.

Next we introduce some lemmas needed in the proofs of the main results of this paper.

Lemma 2.1 ([2]) Let $F$ and $G$ be two nonconstant meromorphic functions sharing $(1,0)$ and $H \neq 0$. Then

$$
\begin{aligned}
T(r, F) \leq & N_{2}\left(r, \frac{1}{F}\right)+N_{2}\left(r, \frac{1}{G}\right)+N_{2}(r, F)+N_{2}(r, G)+2 \bar{N}\left(r, \frac{1}{F}\right) \\
& +\bar{N}\left(r, \frac{1}{G}\right)+2 \bar{N}(r, F)+\bar{N}(r, G)+S(r, F)+S(r, G)
\end{aligned}
$$

and the same inequality holds for $T(r, G)$.

Lemma 2.2 ([2]) Let $F$ and $G$ be two nonconstant meromorphic functions sharing $(1,1)$ and $H \neq 0$. Then

$$
\begin{aligned}
T(r, F) \leq & N_{2}\left(r, \frac{1}{F}\right)+N_{2}\left(r, \frac{1}{G}\right)+N_{2}(r, F)+N_{2}(r, G)+\frac{1}{2} \bar{N}\left(r, \frac{1}{F}\right)+\frac{1}{2} \bar{N}(r, F) \\
& +S(r, F)+S(r, G)
\end{aligned}
$$

and the same inequality holds for $T(r, G)$. 
Lemma 2.3 ([12]) Let $f$ and $g$ be two nonconstant meromorphic functions sharing $(1,2)$. Then one of the following cases holds:

(i) $T(r) \leq N_{2}\left(r, \frac{1}{f}\right)+N_{2}\left(r, \frac{1}{g}\right)+N_{2}(r, f)+N_{2}(r, g)+S(r)$,

(ii) $f=g$,

(iii) $f g=1$, where $T(r)=\max \{T(r, f), T(r, g)\}$ and $S(r)=o\{T(r)\}$, as $r \notin E$, where $E \subset(0,+\infty)$ is a subset of finite linear measure.

Lemma 2.4 ([7]) Let $f$ and $g$ be two meromorphic functions, and let $k$ be a positive integer. If $E_{k}(1 ; f)=E_{k}(1 ; g)$, then one of the following cases must occur:

(i)

$$
\begin{aligned}
T(r, f) & +T(r, g) \\
\leq & N_{2}\left(r, \frac{1}{f}\right)+N_{2}\left(r, \frac{1}{g}\right)+N_{2}(r, f)+N_{2}(r, g) \\
& +\bar{N}\left(r, \frac{1}{f-1}\right)+\bar{N}\left(r, \frac{1}{g-1}\right)-N_{11}\left(r, \frac{1}{f-1}\right)+\overline{N_{(k+1}}\left(r, \frac{1}{f-1}\right) \\
& +\overline{N_{(k+1}}\left(r, \frac{1}{g-1}\right)+S(r, f)+S(r, g) ;
\end{aligned}
$$

(ii) $f=\frac{(b+1) g+(a-b-1)}{b g+(a-b)}$, where $a(\neq 0), b$ are two constants.

Lemma 2.5 ([21]) Letf be a meromorphic function. If

$$
g=\frac{a f+b}{c f+d}
$$

where $a, b, c, d \in S(f)$ and $a d-b c \neq 0$, then $T(r, g)=T(r, f)+S(r, f)$.

Lemma 2.6 ([4]) Let $f$ be a nonconstant meromorphic function, let $\varepsilon>0$ and let $c \in \mathbb{C}$. If $f$ is of finite order, then there exists a set $E=E(f, \varepsilon) \subset(0,+\infty)$ satisfying

$$
\limsup _{r \rightarrow \infty} \frac{\int_{E \cap[1, r)} \frac{d t}{t}}{\log r} \leq \varepsilon,
$$

i.e., of logarithmic density at most $\varepsilon$, such that

$$
m\left(r, \frac{f(z+c)}{f(z)}\right)=O\left(\frac{\log r}{r} T(r, f(z))\right)
$$

for all out of the set $E$. If $\rho_{2}=\rho_{2}<1$ and $\varepsilon>0$, then

$$
m\left(r, \frac{f(z+c)}{f(z)}\right)=o\left(\frac{T(r, f(z))}{r^{1-\rho_{2}-\varepsilon}}\right)
$$

for all $r \in(0,+\infty)$ outside of a set of finite logarithmic measure.

Lemma 2.7 ([1, Lemma 2.3]) Let $k$ be a positive integer, and let $f$ be a nonconstant meromorphic function such that $f^{(k+1)} \not \equiv 0$. If $\bar{N}\left(r, \frac{1}{f}\right)=S(r, f)$, then

$$
k N_{1)}(r, f(z)) \leq \bar{N}_{(2}(r, f)+N_{1)}\left(r, \frac{1}{f^{(k)}-1}\right)+\bar{N}\left(r, \frac{1}{f^{(k+1)}}\right)+S(r, f) .
$$


Lemma 2.8 ([24]) Let $f$ be a meromorphic function such that $f^{(k)}$ is not constant. Then

$$
T(r, f) \leq N\left(r, \frac{1}{f}\right)+N_{1)}\left(r, \frac{1}{f^{(k)}-1}\right)+\bar{N}(r, f)-N\left(r, \frac{1}{f^{(k+1)}}\right)+S(r, f) .
$$

Lemma 2.9([24]) Letf be a transcendental meromorphic function. Then, for each positive real number $\varepsilon$ and for each positive integer $n$,

$$
(n-1) \bar{N}(r, f) \leq(1+\varepsilon) N\left(r, \frac{1}{f^{(n)}}\right)+(1+\varepsilon)(N(r, f)-\bar{N}(r, f))+S(r, f) .
$$

Lemma 2.10 ([4]) Let $f$ be a nonconstant meromorphic function of finite order and $c \in \mathbb{C}$.

Then

$$
\begin{aligned}
& T(r, f(z+c)=T(r, f)+S(r, f), \\
& N\left(r, f(z+c)=N(r, f)+S(r, f), \quad N\left(r, \frac{1}{f(z+c)}\right)=N\left(r, \frac{1}{f(z)}\right)+S(r, f)\right.
\end{aligned}
$$

and

$$
\bar{N}\left(r, f(z+c)=\bar{N}(r, f)+S(r, f), \quad \bar{N}\left(r, \frac{1}{f(z+c)}\right)=\bar{N}\left(r, \frac{1}{f(z)}\right)+S(r, f) .\right.
$$

\section{Proof of Theorem 1.1}

Set $F=\frac{f^{n}}{R}, G=\frac{g^{n}}{R}$. Clearly, $F$ and $G$ share $(1, l)$. Write $T(r)=\max \{T(r, f(z)), T(r, g(z))\}$ and $S(r)=o\{T(r)\}$ as $r \notin E$ and $r \rightarrow \infty$, where $E \subset(0,+\infty)$ is a subset of finite linear measure.

Case $1 . l=0$ and $n \geq 15$.

Assume that $H \neq 0$. By Lemma 2.1, we have

$$
\begin{aligned}
T(r, F) \leq & N_{2}\left(r, \frac{1}{F}\right)+N_{2}\left(r, \frac{1}{G}\right)+N_{2}(r, F)+N_{2}(r, G)+2 \bar{N}\left(r, \frac{1}{F}\right) \\
& +\bar{N}\left(r, \frac{1}{G}\right)+2 \bar{N}(r, F)+\bar{N}(r, G)+S(r, F)+S(r, G) .
\end{aligned}
$$

Clearly,

$$
\begin{aligned}
n T(r, f) & \leq 4 \bar{N}\left(r, \frac{1}{F}\right)+3 \bar{N}\left(r, \frac{1}{G}\right)+4 \bar{N}(r, F)+3 \bar{N}(r, G)+S(r, F)+S(r, G) \\
& \leq 4 \bar{N}\left(r, \frac{1}{f}\right)+3 \bar{N}\left(r, \frac{1}{g}\right)+4 \bar{N}(r, f)+3 \bar{N}(r, g)+S(r, f)+S(r, g) \\
& \leq 8 T(r, f)+6 T(r, g)+S(r, f)+S(r, g) .
\end{aligned}
$$

Similarly, we have

$$
n T(r, g) \leq 8 T(r, g)+6 T(r, f)+S(r, f)+S(r, g) .
$$

Now (1) and (2) yield

$$
(n-14)(T(r, f)+T(r, g)) \leq S(r, f)+S(r, g) .
$$


Hence

$$
(n-14) T(r) \leq S(r),
$$

which contradicts $n \geq 15$.

Therefore, $H \equiv 0$ and we have

$$
\left(\frac{F^{\prime \prime}}{F^{\prime}}-\frac{2 F^{\prime}}{F-1}\right)-\left(\frac{G^{\prime \prime}}{G^{\prime}}-\frac{2 G^{\prime}}{G-1}\right)=0
$$

It follows from (3) that

$$
\frac{1}{F-1}=\frac{A}{G-1}+B
$$

where $A \neq 0, B$ are constants.

Subcase 1.1. If $B=0$, then (4) leads to $F=\frac{G-1+A}{A}$ and $G=A F-(A-1)$. Suppose that $A=1$. Clearly, we have $F=G$, and thus $f=\operatorname{tg}$, where $t^{n}=1$. If $A \neq 1$, then we have $\bar{N}\left(r, \frac{1}{G}\right)=$ $\bar{N}\left(r, \frac{1}{F-\frac{A-1}{A}}\right)$ and $\bar{N}\left(r, \frac{1}{F}\right)=\bar{N}\left(r, \frac{1}{G+(A-1)}\right)$. By Nevanlinna's second fundamental theorem,

$$
\begin{aligned}
T(r, F) & \leq \bar{N}\left(r, \frac{1}{F}\right)+\bar{N}\left(r, \frac{1}{F-\frac{A-1}{A}}\right)+\bar{N}(r, F)+S(r, F) \\
& \leq \bar{N}\left(r, \frac{1}{F}\right)+\bar{N}\left(r, \frac{1}{G}\right)+\bar{N}(r, F)+S(r, F)
\end{aligned}
$$

Thus,

$$
\begin{aligned}
n T(r, f) & \leq \bar{N}\left(r, \frac{1}{f}\right)+\bar{N}(r, g)+\bar{N}(r, f)+S(r, f) \\
& \leq 2 T(r, f)+T(r, g)+S(r, f) .
\end{aligned}
$$

Similarly, we have

$$
n T(r, g) \leq 2 T(r, g)+T(r, f)+S(r, g) \text {. }
$$

Combining (7) and (8), we obtain

$$
(n-3)(T(r, f)+T(r, g)) \leq S(r, f)+S(r, g) \text {. }
$$

Clearly, it is a contradiction by $n \geq 15$.

Subcase $1.2 B \neq 0$.

If $A \neq B$, then we have $F=\frac{(B+1) G-(B-A+1)}{B G+(A-B)}$. Applying Nevanlinna's second fundamental theorem to $F$ with considering 0 point, 1 point, and $\infty$ point, we can also get a contradiction by similar discussion as in Subcase 1.1 .

Case 2. $l=1$ and $n \geq 10$.

Assume that $H \neq 0$. By Lemma 2.2., we have

$$
T(r, F) \leq N_{2}\left(r, \frac{1}{F}\right)+N_{2}\left(r, \frac{1}{G}\right)+N_{2}(r, F)+N_{2}(r, G)+\frac{1}{2} \bar{N}\left(r, \frac{1}{F}\right)+\frac{1}{2} \bar{N}(r, F)
$$




$$
\begin{aligned}
& +S(r, F)+S(r, G) \\
\leq & \frac{5}{2} \bar{N}\left(r, \frac{1}{f(z)}\right)+2 \bar{N}\left(r, \frac{1}{g(z)}\right)+\frac{5}{2} \bar{N}(r, f)+2 \bar{N}(r, g)+S(r, f)+S(r, g) .
\end{aligned}
$$

Thus,

$$
n T(r, f) \leq 5 T(r, f)+4 T(r, g)+S(r, f)+S(r, g) .
$$

Similarly, we have

$$
n T(r, g) \leq 5 T(r, g)+4 T(r, f)+S(r, f)+S(r, g) \text {. }
$$

Combining (5) and (6) yields

$$
(n-9)(T(r, f)+T(r, g)) \leq S(r, f)+S(r, g),
$$

which contradicts $n \geq 10$.

Therefore, $H \equiv 0$. We can deduce the same conclusion by similar discussion as in Case 1 .

Case 3. $l \geq 2$ and $n \geq 9$.

Subcase 3.1. $l=2$.

From Lemma 2.3, if (i) holds, then we deduce that

$$
\begin{aligned}
& \max \{T(r, F), T(r, G)\} \\
& \quad \leq N_{2}\left(r, \frac{1}{F}\right)+N_{2}\left(r, \frac{1}{G}\right)+N_{2}(r, F)+N_{2}(r, G)+S(r, F)+S(r, G) .
\end{aligned}
$$

That is,

$$
\begin{aligned}
n T(r) & =n \max \{T(r, F), T(r, G)\} \leq 2 \bar{N}\left(r, \frac{1}{f}\right)+2 \bar{N}\left(r, \frac{1}{g}\right)+2 \bar{N}(r, f)+2 \bar{N}(r, g)+S(r) \\
& \leq 8 T(r)+S(r) .
\end{aligned}
$$

Therefore, $(n-8) T(r) \leq S(r)$, which contradicts $n \geq 9$. Thus we have $F=G$ or $F G=1$. If $F=G$, then $f^{n}=g^{n}$, which yields $f=t g$, where $t^{n}=1$. If $F G=1$, then $(f g)^{n}=(R)^{2}$, which yields $f g=t \alpha$, where $t^{n}=1, \alpha^{n}=R^{2}$.

Subcase 3.2. $l \geq 3$.

By Lemma 2.4, either (i) or (ii) holds. If (i) holds, then we obtain

$$
\begin{aligned}
T(r, F) & +T(r, G) \\
\leq & N_{2}\left(r, \frac{1}{F}\right)+N_{2}\left(r, \frac{1}{G}\right)+N_{2}(r, F)+N_{2}(r, G) \\
& +\bar{N}\left(r, \frac{1}{F-1}\right)+\bar{N}\left(r, \frac{1}{G-1}\right)-N_{11}\left(r, \frac{1}{F-1}\right)+\overline{N_{(k+1}}\left(r, \frac{1}{F-1}\right) \\
& +\overline{N_{(k+1}}\left(r, \frac{1}{G-1}\right)+S(r, F)+S(r, G) \\
\leq & N_{2}(r, F)+N_{2}(r, G)+N_{2}\left(r, \frac{1}{F}\right)+N_{2}\left(r, \frac{1}{G}\right)+\frac{1}{2} \bar{N}\left(r, \frac{1}{F-1}\right)+\frac{1}{2} \bar{N}\left(r, \frac{1}{G-1}\right)
\end{aligned}
$$




$$
\begin{aligned}
& +S(r, F)+S(r, G) \\
\leq & N_{2}(r, F)+N_{2}(r, G)+N_{2}\left(r, \frac{1}{F}\right)+N_{2}\left(r, \frac{1}{G}\right)+T(r, F)+T(r, G)+S(r, F)+S(r, G) .
\end{aligned}
$$

Therefore, we get

$$
\begin{aligned}
\frac{1}{2} T(r, F)+\frac{1}{2} T(r, G) \leq & 2 \bar{N}(r, f)+2 \bar{N}(r, g)+2 \bar{N}\left(r, \frac{1}{f}\right)+2 \bar{N}\left(r, \frac{1}{g}\right) \\
& +S(r, f)+S(r, g) .
\end{aligned}
$$

Consequently,

$$
\frac{1}{2} n\{T(r, f)+T(r, g)\} \leq 4\{T(r, f)+T(r, g)\}+S(r, f)+S(r, g)
$$

which leads to

$$
\left(\frac{1}{2} n-4\right)\{T(r, f)+T(r, g)\} \leq S(r, f)+S(r, g) .
$$

This is a contradiction since $n \geq 9$.

Hence, (ii) holds, which means

$$
F=\frac{(b+1) G+(a-b-1)}{b G+(a-b)},
$$

where $a \neq 0, b$ are constants.

Suppose that $b=0$. Then we have $F=G$ when $a-1=0$, that is, $f=t g$, where $t^{n}=1$. If $a-1 \neq 0$, then we obtain $F=\frac{G+a-1}{a}$ and $G=a\left(F+\frac{1-a}{a}\right)$, and so $\bar{N}\left(r, \frac{1}{F}\right)=\bar{N}\left(r, \frac{1}{G+a-1}\right)$, $\bar{N}\left(r, \frac{1}{G}\right)=\bar{N}\left(r, \frac{1}{F+\frac{1-a}{a}}\right)$. By Nevanlinna's second fundamental theorem we get

$$
T(r, G) \leq \bar{N}\left(r, \frac{1}{G}\right)+\bar{N}\left(r, \frac{1}{G+a-1}\right)+\bar{N}(r, G)+S(r, G)
$$

This yields

$$
n T(r, g) \leq 2 T(r, g)+T(r, f)+S(r, f) .
$$

Similarly, we have

$$
n T(r, f) \leq 2 T(r, f)+T(r, g)+S(r, f) .
$$

Thus we obtain $(n-3)(T(r, f)+T(r, g)) \leq S(r, f)+S(r, g)$, which is a contradiction with $n \geq 9$. Suppose that $b=-1$. If $a+1=0$, then $F \cdot G \equiv 1$. Hence $f \cdot g=t \alpha$, where $t^{n}=1$, $\alpha^{n}=R^{2}$. If $a+1 \neq 0$, similarly to above, then we can obtain a contradiction. Suppose $b \neq 0$ and $b \neq-1$. By a similar reasoning to the case $b=0$, we can also obtain a contradiction.

This completes the proof of Theorem 1.1. 


\section{Proof of Theorem 1.2}

Without loss of any generality, we can assume $a_{1}=0, a_{2}=1$. Since $\Delta f(z), f^{(k)}$ share $0 \mathrm{CM}$, we have

$$
\frac{\Delta f}{f^{(k)}}=e^{Q}
$$

where $Q$ is a polynomial.

Since the period of $f$ is $c \in \mathbb{C} \backslash\{0\}$, we have $e^{Q(z)}=e^{Q(z+\eta)}$.

Consequently, $e^{Q(z+\eta)-Q(z)}=1$, which leads to $Q^{\prime}(z+\eta)=Q^{\prime}(z)$. Then $Q^{\prime}(z)$ has a period $\eta$ and $Q^{\prime}(z)$ must be a constant.

Now write

$$
Q(z)=a z+b,
$$

where $a, b$ are constants.

Since $\Delta f, f^{(k)}$ share $1 I M$, we get

$$
\frac{\Delta f-1}{f^{(k)}-1}=\alpha(z)
$$

where $\alpha$ is a meromorphic function.

By (11), (12) and $Q(z)=a z+b$, we deduce

$$
\alpha=\frac{f^{(k)} e^{a z+b}-1}{f^{(k)}-1} .
$$

By Lemma 2.5, we obtain

$$
T(r, \alpha)=T\left(r, f^{(k)}\right)+S(r, f)=(k+1) T(r, f)+S(r, f) .
$$

Now we estimate the number of zeros, poles of $\alpha$. From the assumption that $\mu(f)>1$, we know that $T\left(r, e^{Q}\right)=S(r, f)$.

Since $\Delta f, f^{(k)}$ share $1 I M$, it follows from (13) that the zero of $\Delta f(z)-1$ and $f^{(k)}-1$ must be the zero of $e^{a z+b}-1$. Noting that $f^{(k)}-1$ have the same poles with $f^{(k)} e^{a z+b}-1$, then, by (12), we have

$$
\bar{N}(r, \alpha)=\bar{N}\left(r, \frac{1}{f^{(k)}-1}\right) \leq \bar{N}\left(r, \frac{1}{e^{a z+b}-1}\right)=S(r, f)
$$

and

$$
\bar{N}\left(r, \frac{1}{\alpha}\right)=\bar{N}\left(r, \frac{1}{\Delta f-1}\right) \leq \bar{N}\left(r, \frac{1}{e^{a z+b}-1}\right)=S(r, f) .
$$

Therefore, from the Nevanlinna second fundamental theorem, we obtain

$$
T(r, \alpha) \leq \bar{N}(r, \alpha)+\bar{N}\left(r, \frac{1}{\alpha}\right)+\bar{N}\left(r, \frac{1}{\alpha-e^{Q}}\right)+S(r, \alpha) .
$$


Combining (11) and (12), we may write $\alpha-e^{Q}=\frac{\Delta f-f^{(k)}}{\left(f^{(k)}-1\right) f^{(k)}}=\frac{e^{Q}-1}{f^{(k)}-1}$. Then, by (15), we conclude that

$$
T(r, \alpha) \leq 3 \bar{N}\left(r, \frac{1}{e^{a z+b}-1}\right)+S(r, f)=S(r, f)
$$

It contradicts (14).

Therefore, $\Delta f=f^{(k)}$. This completes the proof of Theorem 1.2.

\section{Proof of Theorem 1.3}

Some ideas of our proof come from [1,26]. Without loss of generality, we assume that $f(z+c)$ and $f^{(k)}(z)$ share $1 \mathrm{CM}$ and $\infty \mathrm{IM}$, and $N\left(r, \frac{1}{f}\right)=S(r, f)$. For the general case, we take the transformation $T(z)=\frac{z-a_{3}}{z-a_{1}} \frac{a_{2}-a_{1}}{a_{2}-a_{3}}$, and so $T\left(a_{1}\right)=\infty, T\left(a_{2}\right)=1, T\left(a_{3}\right)=0$. Suppose that $f(z+c) \not \equiv f^{(k)}(z)$. Set

$$
\begin{aligned}
G(z) & =\frac{1}{f(z+c)}\left(\frac{f^{(k+1)}(z)}{f^{(k)}(z)-1}-\frac{f^{\prime}(z+c)}{f(z+c)-1}\right) \\
& =\frac{f^{(k)}(z)}{f(z+c)}\left(\frac{f^{(k+1)}(z)}{f^{(k)}(z)-1}-\frac{f^{(k+1)}(z)}{f^{(k)}(z)}\right)-\left(\frac{f^{\prime}(z+c)}{f(z+c)-1}-\frac{f^{\prime}(z+c)}{f(z+c)}\right) .
\end{aligned}
$$

It follows from the lemma of the logarithmic derivative, Lemma 2.6 and $(18)$ that $m(r, G)=$ $S(r, f)$.

By (17), we see that the possible poles of $G$ can occur at the zeros of $f(z+c)$, the 1 points of $f(z+c)$ and $f^{(k)}(z)$, and the poles of $f(z+c)$ and $f^{(k)}(z)$. If $z_{0}$ is a 1 point of $f(z+c)$, then by a short calculation with Laurent series and (17) we see that $G(z)$ is analytic at $z_{0}$. Since $f(z+c)$ and $f^{(k)}(z)$ share $1 \mathrm{CM}$, we know the 1 points of $f(z+c)$ and $f^{(k)}(z)$ are not the poles of $G(z)$. If $f(z+c)$ has a pole $z_{0}$ with multiplicity $p(\geq 1)$, we need to consider two cases: (i) $z_{0}$ is also a pole of $f^{(k)}(z)$, then by (17) $G(z)=O\left(\left(z-z_{0}\right)^{p-1}\right)$; (ii) $z_{0}$ is not a pole of $f^{(k)}(z)$, and hence $z_{0}$ is not a pole of $f^{(k+1)}(z)$. Then we also have $G(z)=O\left(\left(z-z_{0}\right)^{p-1}\right)$. Similarly, the poles of $f^{(k)}(z)$ are not also the poles of $G(z)$. Therefore, the poles of $F$ can only occur at the zeros of $f(z+c)$. By Lemma 2.7 and the hypothesis of Theorem 1.3, it follows that $N\left(r, \frac{1}{f(z+c)}\right)=N\left(r, \frac{1}{f}\right)+S(r, f)=S(r, f)$, and so we have $N(r, G)=S(r, f)$. Thus,

$$
T(r, G)=S(r, f) .
$$

If $G \equiv 0$, then, by (17), we find that $f^{(k)}(z)-1=t(f(z+c)-1)$, with $t \neq 0$ constant. Thus, $(1-t) m\left(r, \frac{1}{f}\right) \leq m\left(r, \frac{f^{(k)}(z)}{f(z)}\right)+m\left(r, \frac{f(z+c)}{f(z)}\right)=S(r, f)$. Since $N\left(r, \frac{1}{f}\right)=S(r, f)$, we have $T\left(r, \frac{1}{f}\right)=$ $S(r, f)$. It is a contradiction. Then $G \not \equiv 0$. And so we deduce from (17) and (19) that

$$
\begin{aligned}
m(r, f(z+c)) & \leq m\left(r, \frac{1}{G}\right)+m\left(r, \frac{f^{(k+1)}(z)}{f^{(k)}(z)-1}-\frac{f^{\prime}(z+c)}{f(z+c)-1}\right) \leq T(r, G)+S(r, f) \\
& =S(r, f) .
\end{aligned}
$$

If $z_{0}$ is a pole of $f(z+c)$ of multiplicity $p \geq 2$, then by (17) we know that $z_{0}$ is possible a zero of $G$ with multiplicity $p-1$. Consequently, it follows from (19) that

$$
N_{(2}(r, f(z+c)) \leq 2 N\left(r, \frac{1}{G}\right) \leq 2 T(r, G)+O(1)=S(r, f) .
$$


Let $z_{0}$ be a simple pole of $f(z+c)$. Set

$$
H(z)=\frac{f^{(k+1)}(z)(f(z+c)-1)}{f^{\prime}(z+c)\left(f^{(k)}(z)-1\right)} .
$$

By a short calculation with Laurent series, it follows that $H\left(z_{0}\right)=k+1$. If $H(z) \equiv k+1$, then we have $f^{(k)}(z)-1=t(f(z+c)-1)^{k+1}$ with $t \neq 0$ constant. This a contradiction, since $f^{(k)}(z)$ and $f(z+c)$ share $1 \mathrm{CM}$. Thus $H \not \equiv k+1$, and so

$$
N_{1)}(r, f(z+c)) \leq N\left(r, \frac{1}{H-(k+1)}\right) \leq T(r, H)+O(1) .
$$

We now estimate the poles of $H$. Clearly, the poles of $H$ can only occur at the 1 points of $f^{(k)}(z)$, the zeros of $f^{\prime}(z+c)$, and the poles of $f(z+c)$ and $f^{(k+1)}(z)$. Since $f^{(k)}(z), f(z+c)$ share $1 \mathrm{CM}$ and $\infty \mathrm{IM}, H$ is holomorphic at the 1 points of $f^{(k)}(z)$ and the poles of $f(z+c)$ and $f^{(k+1)}(z)$. Thus

$$
N(r, H) \leq N_{0}\left(r, \frac{1}{f^{\prime}(z+c)}\right)+S(r, f)
$$

where $N_{0}\left(r, \frac{1}{f^{\prime}(z+c)}\right)$ denotes the zeros of $f^{\prime}(z+c)$ which are not zeros of $f(z+c)-1$. Again by (22), we see that

$$
m(r, H)=S(r, f)
$$

from this, (23) and (24) we find that

$$
N_{1)}(r, f(z+c)) \leq N_{0}\left(r, \frac{1}{f^{\prime}(z+c)}\right)+S(r, f)
$$

Combining this, Nevanlinna's second fundamental theorem (see [10, Theorem 3.2])for $f(z+c),(21)$ and the hypothesis $N\left(r, \frac{1}{f(z)}\right)=S(r, f)$ we have

$$
\begin{aligned}
T(r, f(z+c)) \leq & N\left(r, \frac{1}{f(z+c)}\right)+\bar{N}\left(r, \frac{1}{f(z+c)-1}\right)+\bar{N}(r, f(z+c)) \\
& -N_{0}\left(r, \frac{1}{f^{\prime}(z+c)}\right)+S(r, f(z+c)) \\
\leq & \bar{N}\left(r, \frac{1}{f(z+c)-1}\right)+S(r, f) \\
\leq & N_{1)}\left(r, \frac{1}{f(z+c)-1}\right)+\bar{N}_{(2}\left(r, \frac{1}{f(z+c)-1}\right)+S(r, f) .
\end{aligned}
$$

From this and Nevanlinna's first fundamental theorem, it is easy to deduce that

$$
m\left(r, \frac{1}{f(z+c)-1}\right)+N_{(2}\left(r, \frac{1}{f(z+c)-1}\right) \leq \bar{N}_{(2}\left(r, \frac{1}{f(z+c)-1}\right)+S(r, f),
$$

which implies

$$
m\left(r, \frac{1}{f(z+c)-1}\right)+N_{(2}\left(r, \frac{1}{f(z+c)-1}\right)=S(r, f) .
$$


From (29), we see that

$$
\begin{aligned}
T(r, f(z+c))= & m\left(r, \frac{1}{f(z+c)-1}\right)+N_{1)}\left(r, \frac{1}{f(z+c)-1}\right)+N_{(2}\left(r, \frac{1}{f(z+c)-1}\right) \\
& +S(r, f) \\
= & N_{1)}\left(r, \frac{1}{f(z+c)-1}\right)+S(r, f) .
\end{aligned}
$$

Since $f(z+c)$ and $f^{(k)}(z)$ share $1 \mathrm{CM}$, it follows from Lemma 2.7 and (21) that

$$
(k-1) N_{1)}(r, f(z+c)) \leq \bar{N}\left(r, \frac{1}{f^{(k+1)}(z)}\right)+S(r, f) .
$$

By Lemma 2.8, (29) (30) and (21), we have

$$
\begin{aligned}
N\left(r, \frac{1}{f^{(k+1)}(z+c)}\right) \leq & \left.N\left(r, \frac{1}{f(z+c}\right)+N_{1)}(r, f(z+c))\right)+N\left(r, \frac{1}{f(z+c)-1}\right) \\
& -T(r, f(z+c))+S(r, f(z+c)) \\
\leq & N_{1)}(r, f(z+c))+S(r, f) .
\end{aligned}
$$

It follows from Lemma 2.10 that

$$
N\left(r, \frac{1}{f^{(k+1)}(z)}\right)=N\left(r, \frac{1}{f^{(k+1)}(z+c)}\right)+S(r, f) .
$$

From this, (31) and (32), we see that $(k-2) N_{1)}(r, f(z+c))=S(r, f)$. If $k \geq 3$, then $N_{1)}(r, f(z+$ $c))=S(r, f)$. Combining this, (20) and (21), we have $T(r, f(z+c))=S(r, f)$, which is a contradiction.

Let $k=2$.

Case 1. If $f(z)$ is transcendental, then, by Lemma 2.9, for a positive constant $\varepsilon<1$ we have

$$
\begin{aligned}
2 N_{1)}(r, f(z+c)) \leq & (1+\varepsilon) N\left(r, \frac{1}{f^{(k+1)}(z+c)}\right)+(1+\varepsilon)[N(r, f(z+c))-\bar{N}(r, f(z+c))] \\
& +S(r, f) .
\end{aligned}
$$

From this and (21) we have

$$
(1-\varepsilon) N_{1)}(r, f(z+c))=S(r, f)
$$

Combining this, (20) and (21), we have $T(r, f(z+c))=S(r, f)$. This is a contradiction.

Case 2. If $f(z)$ is rational, then by $N\left(r, \frac{1}{f}\right)=S(r, f)$ we know that $f$ has no zeros, and hence we can write $f(z+c)=\frac{1}{P(z)}$, where $P(z)$ is a nonconstant polynomial. Set $\Phi=\frac{f^{(k+1)}(z)}{f^{(k)}(z)-1}-$ $\frac{f^{\prime}(z+c)}{f(z+c)-1}-2 \frac{f^{\prime}(z+c)}{f(z+c)}$. Clearly $T(r, \Phi)=S(r, f)$. Combining this and (17), we have

$$
2 f^{\prime}(z+c)=G f^{2}-\Phi f .
$$


Substituting $f(z+c)=\frac{1}{P(z)}$ into (34) we obtain $-2 P^{\prime}=G-\Phi P$. This shows that $T\left(r, P^{\prime}\right)=$ $T(r, P)+S(r, f)$ and so $T(r, P)=S(r, f)$. This is a contradiction.

Therefore, $f(z+c)=f^{(k)}(z)$. This completes the proof of Theorem 1.3.

\section{Acknowledgements}

The authors are grateful to the referees for their valuable suggestions and comments which have improved the clarity of the paper.

\section{Funding}

The work was supported by NNSF of China (No.11971344).

\section{Availability of data and materials}

Not applicable.

\section{Competing interests}

The authors declare that they have no competing interests.

\section{Authors' contributions}

Each of the authors contributed to each part of this study equally and approved the final version of this manuscript.

\section{Publisher's Note}

Springer Nature remains neutral with regard to jurisdictional claims in published maps and institutional affiliations.

Received: 26 May 2020 Accepted: 31 August 2020 Published online: 09 September 2020

\section{References}

1. Al-Khaladi, A.H.H.: Meromorphic functions that share one finite value CM or IM with their $k$-th derivatives. Results Math. 63, 95-105 (2013)

2. Banerjee, A.: Meromorphic functions sharing one value. Int. J. Math. Math. Sci. 22, 3587-3598 (2005)

3. Chen, B., Li, S., Chen, Z.X.: Uniqueness of difference operators of meromorphic functions. J. Inequal. Appl. 2012, 48 (2012)

4. Chiang, Y.M., Feng, S.J.: On the Nevanlinna characteristic of $f(z+\eta)$ and difference equations in the complex plane. Ramanujan J. 16, 105-129 (2008)

5. Chiu, K.-S., Li, T.: Oscillatory and periodic solutions of differential equations with piecewise constant generalized mixed arguments. Math. Nachr. 292, 2153-2164 (2019)

6. Deng, B.M., Lei, C.L., Fang, M.L.: Meromorphic function sharing sets with its difference operator or shifts. Chin. Ann Math., Ser. B 40(3), 331-338 (2019)

7. Fang, C.Y., Fang, M.L.: Uniqueness of meromorphic functions and differential polynomials. Comput. Math. Appl. 44, 607-617 (2002)

8. Frank, G., Weissenborn, G.: Meromorphic funktionen, die mit einer ihrer Ableitungen Werte teilen. Complex Var. Theory Appl. 7, 33-43 (1986)

9. Halburd, R.G., Korhonen, R.J.: Difference analogue of the lemma on the logarithmic drivative with applications to difference equations. J. Math. Anal. Appl. 314, 477-487 (2006)

10. Hayman, W.K.: Meromorphic Functions, Clarendon, Oxford (1964)

11. Heittokangas, J., Korhonen, R., Laine, I., Rieppo, J., Zhang, J.: Value sharing results for shifts of meromorphic function and sufficient conditions for periodicity. J. Math. Anal. Appl. 355, 352-363 (2009)

12. Lahiri, I.: Weighted sharing and uniqueness of meromorphic functions. Nagoya Math. J. 161(3), 193-206 (2001)

13. Lahiri, I.: Weighted value sharing and uniqueness of meromorphic functions. Complex Var. Theory Appl. 46(3), 241-253 (2001)

14. Li, T., Pintus, N., Viglialoro, G.: Properties of solutions to porous medium problems with different sources and boundary conditions. Z. Angew. Math. Phys. 70, Article ID 86, 1-18 (2019)

15. Nevanlinna, R.: Einige Eindeutigkeitssätze in der Theorie der meromorphen Funktionen. Acta Math. 48, 367-391 (1926)

16. Ozawa, M.: Unicity theorems for entire functions. J. Anal. Math. 30, 411-420 (1976)

17. Qi, X.G., Li, N., Yang, L.Z.: Uniqueness of meromorphic functions concerning their differences and solutions of difference Painleve equations. Comput. Methods Funct. Theory 18, 567-582 (2018)

18. Rubel, L.A., Yang, C.C.: Values shared by an entire function and its derivative. In: Lecture Notes in Math., vol. 599, pp. 101-103. Springer, New York (1977)

19. Ueda, H.: On the zero-one-pole set of a meromorphic function II. Kodai Math. J. 13, 134-142 (1990)

20. Wang, J.: Uniqueness of entire function sharing a small function with its derivative. J. Math. Anal. Appl. 362(2) 387-392 (2010)

21. Yang, C.C., Yi, H.X.: Uniqueness Theory of Meromorphic Functions. Kluwer Academic, Dordrecht (2003)

22. Yi, H.X.: Meromorphic functions that share three values. Chin. Ann. Math. 9, 434-440 (1988)

23. Yi, H.X.: Meromorphic functions that share two or three values. Kodai Math. J. 13, 363-372 (1990)

24. Yi, H.X., Li, X.M.: Meromorphic functions sharing four values. Proc. Jpn. Acad., Ser. A, Math. Sci. 83, 123-128 (2007)

25. Zhang, J.J., Liao, L.W.: Entire functions sharing some values with their difference operators. Sci. China Math. 57, 2143-2152 (2014)

26. Zhang, Q.C.: Uniqueness of meromorphic functions with their derivatives. Acta Math. Sin. 45(9), 871-876 (2002) 